\title{
Anectodal report of acute gastric anisakiasis and severe chest discomfort
}

\author{
Enrica Mariano ${ }^{3}$, Massimo Fioranelli ${ }^{1}$, Maria Grazia Roccia ${ }^{2}$, Maurizio Onorato ${ }^{4}$, Veronica Di Nardo ${ }^{1}$ and Maria Bianchi $^{1}$ \\ ${ }^{1}$ G.Marconi University, Rome, Italy \\ ${ }^{2}$ University B.I.S. Group of Institutions, Punjab Technical University, Punjab, India \\ ${ }^{3}$ Tor Vergata University, Rome Italy \\ ${ }^{4}$ Casa di Cura Mater Dei
}

\section{Introduction}

Anisakiasis is a gastrointestinal infection caused by larvae, mainly of Anisakis species and Pseudoterranova species, found in saltwater fish. Its symptoms, including abdominal pain, nausea, and vomiting, occur usually within 48 hours after ingestion of raw sea water fish, and are caused by penetration of larvae into the gastrointestinal wall. Consideration of this disease based on a history of prior ingestion of raw fish is important for the diagnosis, and a definite diagnosis of gastric anisakiasis is easily made by endoscopic examination. Although usually no specific treatment is needed, relief of the symptoms is hastened by endoscopic removal of larvae. Gastric irritation, such as peptic ulcer disease, may be included in the causes of chest pain or discomfort, but gastric anisakiasis presenting with chest pain has been reported in only a few cases to our knowledge.

\section{Case report}

We report the case of a 53 years-old woman whose clinical history was positive for patent foramen ovale that was admitted in our department for elective percutaneous closure of interatrial a defect associated with huge redundant interatrial aneurysm. Clinical history was negative for cardiovascular risk factors and documented a previous intervention for mammalian neoplasia and multidrugs allergy. The patient suffered from migraine for many years while MRI findings documented hyperintense areas compatible with signs of previous cerebral ischaemia.

Therefore, the patient was subjected to percutanous closure of patent foramen ovale by means of device Amplatzer $\mathrm{n} 35 \mathrm{~mm}$. Procedure was free from significant complications and echocardiographic follow-up showed correct placement of device and no significant interatrial shunt at color-Doppler.

The patient was discharged in optimal clinical conditions with medical therapy based on clopidogrel, aspirin and proton pump inhibitors.

After one week from discharge the patient underwent rehospitalization for intense stomachache associated with aspecific chest pain. Blood pressure, pulse, heart and respiratory parameters were within normal limits.

Serial blood test, electrocardiogram, echocardiography, chest X-ray and abdominal ultrasound documented no significant alterations (Figures 1-3).
Patient experienced worsening of chest constriction symptoms so that the patient was subjected to coronary contrast computed tomography performed four hours later that showed no significant coronary narrowings and good placement of device Amplatzer at the level of fossa ovalis (Figures 4-6).

Finally careful endoscopic gastric examination was performed that revealed a superficial ulcer in the gastric fund wall. A single worm was removed with biopsy forceps and multiple biopsies were perfomed close to the penetration site.

Histologic findings documented nonspecific signs of hyperemia with eosinophilic infiltration and superficial epithelium hyperplasia associated with fragments of white parasite compatible with Anisakis diagnosis. H. pylori morphological and histochemical research was negative.

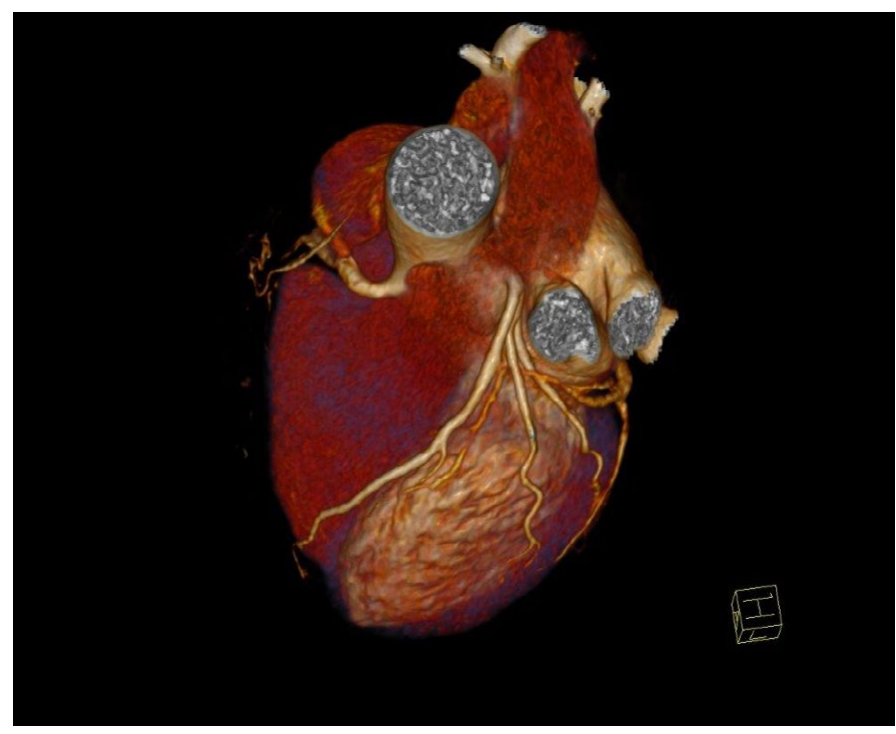

Figure 1. Top. Brain networks associated with mind wandering and attention.

Correspondence to: Prof. Massimo Fioranelli, M.D. Associate Professor of Physiology, Guglielmo Marconi University, Rome, Italy; E-mail: massimo.fioranelli@gmail.com

Received: September 02, 2015; Accepted: September 28, 2015; Published: October 04, 2015 


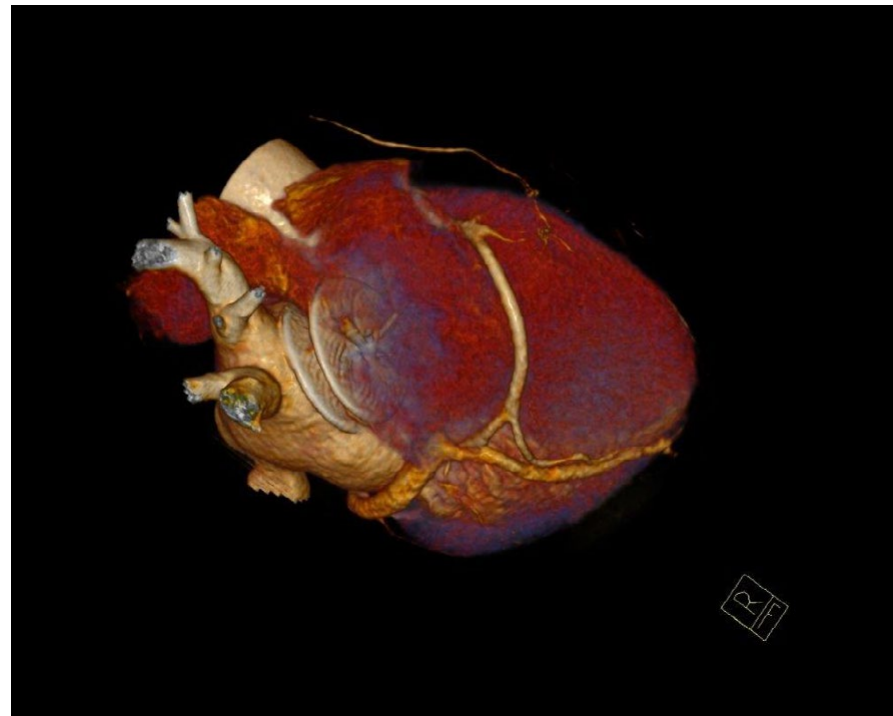

Figure 2. Top. Brain networks associated with mind wandering and attention.

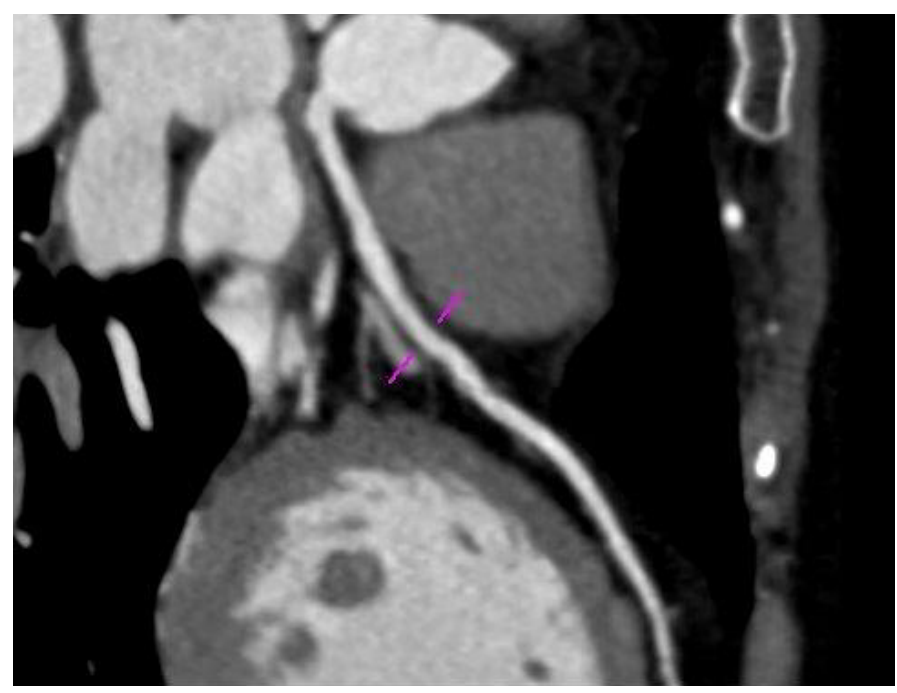

Figure 3. Top. Brain networks associated with mind wandering and attention.

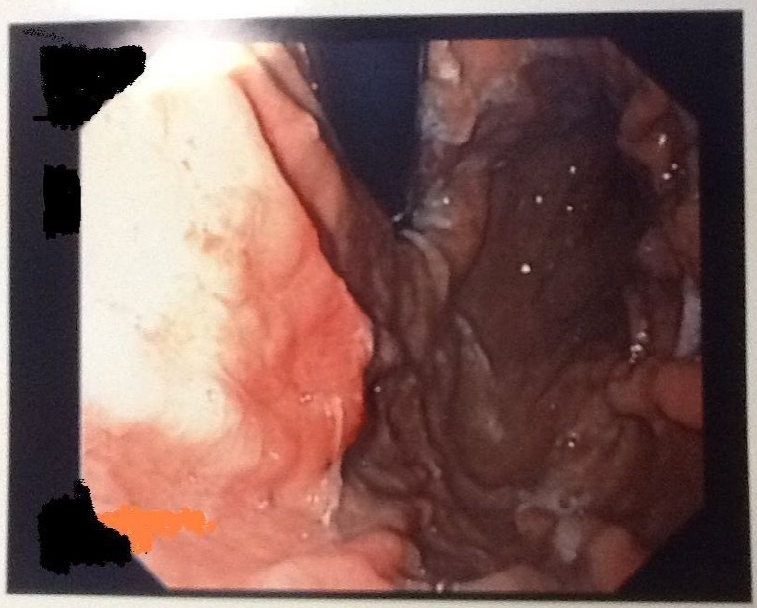

Figure 4. Endoscopy findings showing erosion at fundus-body stomach level
We insisted on asking the patient about the ingestion of undercooked fish and accurate patient history confirmed about the ingestion of marinated anchovies the night before onset of gastric symptoms.

The patient conditions immediately improved after removing the worm. She was discharged after receiving supportive care. The 12-month follow-up was negative for relevant clinical events.

\section{Discussion}

Gastrointestinal anisakias is a parasite infection acquired by infection with Anisakis simplex or Pseudoterranova decipiens nematodes belonging to the Anisakidae family, occurring in people that consume raw or inadequately cooked fish or squid. It is frequently characterized by severe epigastric pain, nausea and vomiting caused by the penetration of the larvae into the gastric wall $[1,2]$.

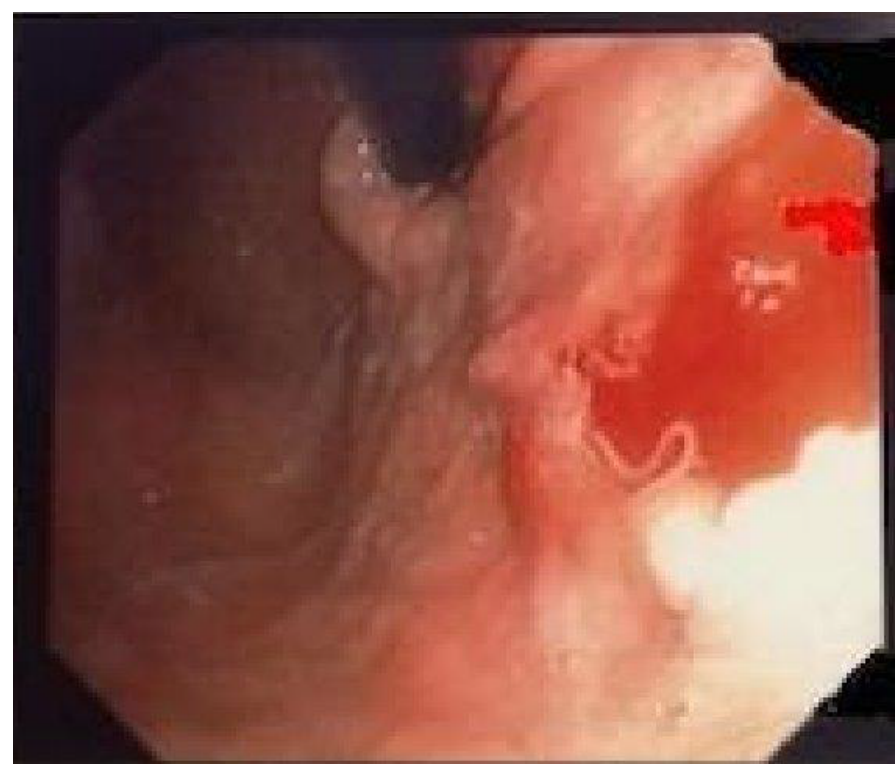

Figure 5. Anisakis finding s during procedural multiple biopsies.

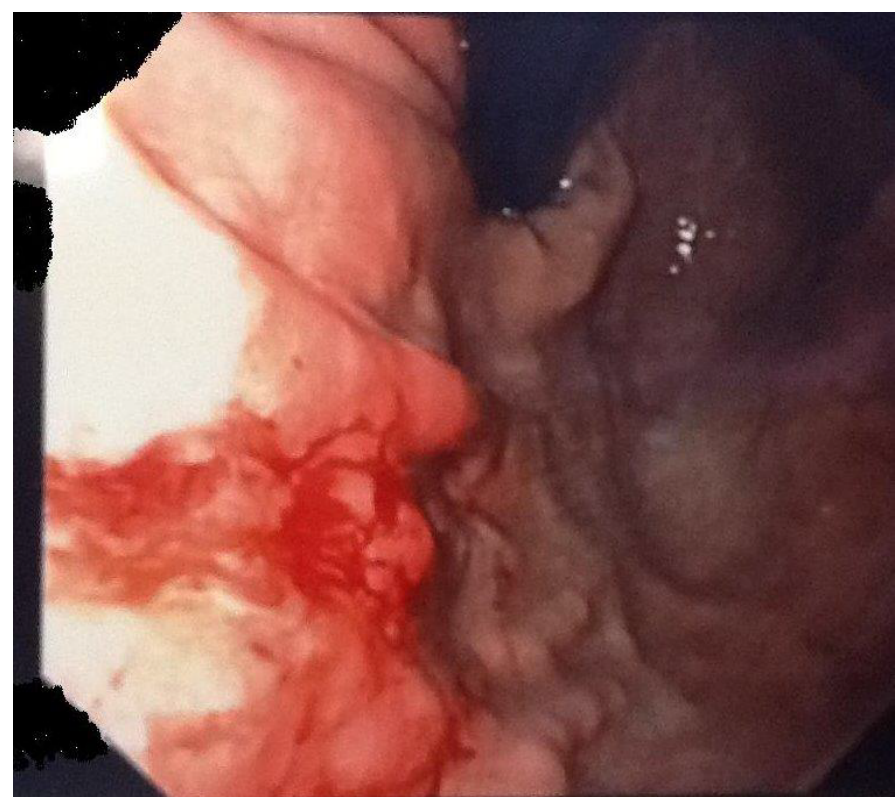

Figure 6. Clear documentation of bleeding erosion after parasite removal. 
Larval anisakias was firstly reported in 1960 by Van Thiel et al. [3]. Humans become infected by ingestion of the As Larvae, which are present in seafood dishes. Many cases have been reported in Japan [4] where there is a high rate of consumption of raw fish but the disease has been recognized in some other countries, especially the Netherlands, Germany, France, and Spain, United States [5-11]. Acute gastric anisakiasis with severe chest discomfort is rarely reported in Italy $[6,7]$. The gastric anisakiasis has increased proportionally with high consumption of sushi, sashimi, raw and smoked fish dishes.

Herring, cod, squid, mackerel, tuna and cuttle fish are the most commonly potential larval conductors.

Four major clinical syndromes are described in infected individuals, including gastric (GA), intestinal, ectopic and allergic diseases [9]. The most common presentation, the GA is frequently characterized by severe epigastric pain, nausea, vomiting and is caused by penetration of the larvae in the gastrointestinal wall. GA (acute form) is severe epigastric pain occurring usually within 12 hours of eating raw fish such as tuna, cuttlefish, shrimp, herring or mackerel. Generally, the diagnosis is made by the endoscopic identification of the anisakis larva.

On the other hand, gastro-allergic anisakiasis with rash, urticaria and isolated angioedema [10,11] or anaphylaxis is a clinical entity that has been described only recently. Also, if patients usually develop symptoms within 12 hours after raw seafood ingestion, not always endoscopic exploration can promptly identify the Anisakis larvae.

In cases where upper endoscopy is performed within few hours or days after ingestion, endoscopic findings show gastric reddening, oedema and erosions or ulcerations around the worm penetration site, which may frequently affect the gastric greater curvature.

Endoscopic examination, when delayed, can highlight a chronic mucosal change with a greater difficulty in anisakis detection. The endoscopic worm extraction is usually followed by a rapid improvement in symptoms while a delayed treatment may allow the larvae to penetrate into the submucosa.

While intestinal or extraintestinal infections can required surgical removal of the larvae, the diagnosis of GA obtained with a noninvasive method allows a conservative treatment by supportive therapy or albendazole administration.

Chest discomfort during anisakias was rarely reported.

On the other hand, with chronic gastric anisakiasis, the larvae are difficult to find at endoscopy, making the diagnosis difficult. A submucosal tumour is one of the characteristic features of chronic gastric anisakiasis. Chronic anisakiasis [1,2] is really rare and its diagnosis is difficult. The diagnosis is often made incidentally at endoscopy or after discovery of a mass in the abdomen. The authors assume that penetration of the anisakis larvae through the gastric mucosa caused formation of an abscess within the muscular layer, with subsequent rupture of the abscess leading to transient perforation.

\section{Conclusions}

In our reported case, the chest discomfort described by our patient was so severe that the patient was admitted in our Emergency Department. Occurring within few hours after parasite ingestion and contact, upper accurate endoscopic examination was effective as diagnostic confirmation and curative treatment considering that the endoscopic removal was followed by rapid improvement in symptoms.

Generally, removal of the larvae at endoscopy is curative for acute gastric anisakiasis and the severe epigastric pain disappears rapidly. On the other hand, If the anisakis larvae cannot be removed, the symptoms continue for a few days until the larvae die within the gastric mucosa $[2,6,11]$.

Sometimes, a significant allergic reaction may have hindered the permanence of the larva in the stomach and its penetration through the gastric wall, thus making diagnosis impossible.

The clinical implication of these considerations is reflected on the patient management. In fact, the gastroallergic anisakiasis clinical manifestations rather than the gastric or "surgical" symptoms may authorize, if this hypothesis is confirmed, to adopt a "wait and see" treatment.

On the other hand, immediate removal of parasite by means of endoscopy allows rapid relieve of symptoms in gastric Anisakiasis as in our case report.

\section{References}

1. Eskesen A, Strand EA, Andersen SN, Rosseland A, Hellum KB, et al. (2001) Anisakiasis presenting as an obstructive duodenal tumor. A Scandinavian case. Scand $J$ Infect Dis 33: 75-76. [Crossref]

2. Céspedes M, Saez A, Rodríguez I, Pinto JM, Rodríguez R (2000) Chronic anisakiasis presenting as a mesenteric mass. Abdom Imaging 25: 548-550. [Crossref]

3. Van Thiel P, Kuipers FC, Roskam RT (1960) A nematode parasitic to herring, causing acute abdominal syndromes in man. Trop Geogr Med 12: 97-113. [Crossref]

4. Ishikura H (1998) General survey of anisakis and anisakis. In: Ishihara H, Namiki $\mathrm{M}$ eds. Anisakiasis in Japan. Epidemiology, Diagnosis, Treatment. Tokyo: SpringerVerlag; 3-11.

5. Kliks MM (1983) Anisakiasis in the western United States: four new case reports from California. Am J Trop Med Hyg 32: 526-532. [Crossref]

6. Pontone S, Leonetti G, Guaitoli E, Mocini R, Manfredelli S, et al. (2012) Should the host reaction to anisakiasis influence the treatment? Different clinical presentations in two cases. Rev Esp Enferm Dig 104: 607-610. [Crossref]

7. Zullo A, Hassan C, Scaccianoce G, Lorenzetti R, Campo SM, et al. (2010) Gastric anisakiasis: do not forget the clinical history! J Gastrointestin Liver Dis 19: 359. [Crossref]

8. Fernández Salazar LI, Guantes de Vigo B, Herreros Rodríguez J, Abril Vega C, Calabia del Campo J, et al. (2010) Another multiple gastric anisakiasis case. Rev Esp Enferm Dig 102: 60-61. [Crossref]

9. Hochberg NS, Hamer DH (2010) Anisakidosis: Perils of the deep. Clin Infect Dis 51 806-812. [Crossref]

10. Kakizoe S, Kakizoe H, Kakizoe K, Kakizoe Y, Maruta M, et al. (1995) Endoscopic findings and clinical manifestation of gastric anisakiasis. Am J Gastroenterol 90: 761763. [Crossref]

11. López-Serrano MC, Gomez AA, Daschner A, Moreno-Ancillo A, de Parga JM, et al. (2000) Gastroallergicanisakiasis: findings in 22 patients. $J$ Gastroenterol Hepatol 15: 503-506. [Crossref]

Copyright: (C2015 Mariano E. This is an open-access article distributed under the terms of the Creative Commons Attribution License, which permits unrestricted use, distribution, and reproduction in any medium, provided the original author and source are credited. 